\title{
Other Papers
}

\section{Experimental clues in favor of a generalized norm of reciprocity}

\begin{abstract}
The norm of reciprocity (Gouldner, 1960) has only been considered and experimentally demonstrated between two individuals. However, comments from several authors invite the consideration of an expanded form of this norm. 120 passersby, randomly assigned to 3 conditions, were asked to watch a confederate's belongings. Depending on the condition, they had either previously been given a gift or not. In addition, the gift was offered by either the confederate making the target request or by a second confederate, not initially involved. First, results show that passersby are significantly more likely to comply with the target request if they were offered a gift than if they weren't no matter who the confederate was. Second, the confederate offering the gift gets significantly more compliance than the one not initially involved. Results are discussed in terms of self-presentation theory (Pendleton \& Batson, 1979), internalization of norm (Schwartz \& Howard, 1981) and positive mood induction (Isen \& Al, 1978).
\end{abstract}

Key words: social responsibility, reciprocity, Helping behavior, Altruism

"One of the key human characteristics is our willingness to help others in need" (Warneken, 2013; p. 431). However, this willingness could be reinforced by contextual elements such as the attribution of success following the completion of a task (Clark \& Waddell, 1986), being smiled at (Solomon, Zener-Solomon, Arnone, Maur, Reda, \& Roth, 1981; Guéguen \& De Gail, 2003; Vrugt \& Vet, 2009), finding coins on the shelf of a phone box or on the ground (Batson, Coke, Chard, Smith, \& Talaferro, 1979; Isen \& Levin, 1972; Levin \& Isen, 1975) or being offered a piece of cake, a drink or help, beforehand (Harris \& Smith, 1975; Isen \& Levin, 1972; Regan, 1971; Berkowitz $\&$ Daniels, 1964). According to the authors' theoretical hypothesis, induced positive mood (Isen, Shalker, Clark, \& Karp, 1978) or the respect of the standard of reciprocity (Gouldner, 1960) or of social responsibility (Berkowitz \& Daniels, 1963) could explain the observed effect. This manuscript focuses specifically on this second set of interpretations by questioning an inconsistency in research.

Proposed by Gouldner (1960) as a universal principle governing the exchanges between individuals, the norm of reciprocity first prescribes the acceptance of a favor from someone and second, the duty to return the favor. Within the framework of a so-called study on artistic judgement, Regan (1971) brought two individuals into a room: a confederate and a naive subject. During the break, the confederate left the room, then based on the condition either returned with two cans of soda and offered one to the participant, or returned empty-handed. At the end of the experiment, he asked the participant to buy some raffle tickets. The results reveal that the preliminary drink offer statistically increases the number of raffle tickets sold. Replications in quasi-identical experimental contexts reveal that the returned favor remains observed in the absence of the solicitor (Burger, Sanchez, Imberi, \& Grande, 2009), or when the latter is unable to know of the participant's actual behavior (Whatley, Webster, Smith, \& Rhodos, 1999), but on condition that the lapse of time separating the initial gift from the final request does not exceed a few hours (Burger, Horita, Kinoshita, Roberts, \& Vera, 1997). On the contrary, the reciprocity effect disappears if the target request is of an antisocial nature (Boster, Fediuk, \& Kotowski,

\footnotetext{
* Université de Bretagne Sud

** IAE Brest

*** Université de Rouen
} 
2001). Furthermore, although the hypothesis was not specifically tested by the authors, the results of Regan (1971), and Burger, Ehrlichman, Raymond, Ishikawa and Sandoval (2006, exp. 3) suggest that the reciprocity effect can be observed only in regard to a single person: the initial donor.

The internalization of the norm (Gouldner, 1960) is the privileged interpretation of the observed effects. In reference to Schwartz and Howard's (1981) model, reciprocity, considered as a personal standard, is assumed to be activated by the preliminary gift and puts the individual under the moral obligation to return what he has received so as not to feel guilty. However, a second explanation underlining not the moral character of the behavior but the consequences in terms of social evaluation has been put forward. In agreement with self-presentation theory (Pendleton and Batson, 1979; Baumeister, 1982), the image that individuals wish to display to others guides their behavior in public. Counter-normative behavior would place the individual in a deviant situation with social disapproval as a consequence. Those who violate the norm of reciprocity are qualified by Cialdini (2004) as "Ungrateful" or "free-loaders" (p. 32). On the contrary, pro-normative behavior would earn the individual a favorable social evaluation.

The norm of reciprocity is classically distinguished from the norm of social responsibility (Berkowitz \& Daniels, 1963). The latter also aims to account for helping behavior but by prescribing that, "People must help those who are dependent upon them" (Berkowitz \& Daniels, 1964, p. 275). Berkowitz and Daniels (1963) invited pairs of individuals - a participant and a confederate acting as a supervisor - to jointly carry out a task. Based on the condition, the participant was either told that his performance would determine the evaluation of the "supervisor" for a possible reward, or the participant was told nothing at all. The results reveal that the relation of induced dependence significantly increases the participant's performance.

However, certain experiments would tend to bring the two norms closer (Staub, 1972). Berkowitz and Daniels (1964), wishing to demonstrate that contextual elements could also activate the norm of social responsibility reproduced their original experiment by asking the participants to first carry out a task supposedly unrelated to the following. During this experiment, a confederate other than the supervisor either offered help to the participant or did not. The results reveal that the preliminary assistance coupled with the relation of dependence with the supervisor significantly increases the participant's performance. Based on the same protocol, Goranson and Berkowitz (1966), also demonstrated that the participant's performance was higher when the confederate's help was presented as spontaneous compared with help that was planned within the protocol and announced in advance, or compared with a refusal to help (help planned in the protocol but refused by the confederate). The participant's performance is nonetheless greater when the initial help comes from the supervising confederate. Finally, results from Greenglass
(1969) reveal that the participant's performance, having benefited from the help of the supervising confederate, is significantly higher when he had been previously helped by a confederate other than the supervisor than when he hadn't been helped (help not planned in the protocol) or when he had been put in a difficult situation by this confederate (help planned and announced in the protocol but refused by the latter). These results are observed whether the supervisor appears similar or dissimilar to the confederate from the first phase (this variable was manipulated through responses obtained from a questionnaire presented to the participant).

To give an account of this effect, the authors specifically evoked the existence of a mechanism distinct from the norm of social responsibility, namely: generalized reciprocity: "Participants helped by the confederate felt indebted [...] This feeling could have been generalized toward the supervisor in that by working hard for this person, they were reciprocating the help they had received" (Berkowitz \& Daniels, 1964, p. 281; Goranson \& Berkowitz, 1966, p. 228). More recently, Dieckman (2004) also refers to a generalized form of reciprocity to account for cooperation, observed under certain conditions, in repeated social dilemmas situations involving several people.

This proposal, which opposes the data of Regan (1971) and Burger and Al (2006, exp. 3) presenting a reciprocity effect essentially aimed at the initial donor, motivated the experimental investigation of a generalized form of reciprocity. One could consider that some attempts to test this hypothesis have been already made in the context of the DITF compliance gaining paradigm - which reciprocity is one of the explaining theories - by using two different requesters. Results are not consistent: some succeeding to obtain an effect in the presence of two requesters (Terrier, Marfaing, \& Boldi, 2013) and some others failing (Cialdini, Vincent, Lewis, Catalan, Wheeler, \& Darby, 1975, exp. 2). The context and more precisely the laps of time between the two requests and the final beneficiary of the request were confounded variables, and conditioned the results (Dillard, Hunter et Burgoon, 1984).

To test directly the generalized reciprocity hypothesis, without activating the norm of social responsibility, we conceived a protocol in which the relationship of dependence between individuals was not explained and the preliminary favor was not an act of assistance. Furthermore, and in order to extend the reach, our investigation was carried out in a natural context among solicited passersby in the street. Based on the results of Goranson and Berkowitz (1966) and Greenglass (1969), we expected to observe a generalized reciprocity effect, however on a smaller scale than that of the classic reciprocity effect. 


\section{Method}

\section{Participants}

120 participants $\left(63\right.$ women and 57 men $^{1}$ ) took part in the experiment. They were directly approached in a pedestrian area in the town center of Lorient in the south of Brittany during the end of the year holidays in 2012.

\section{Procedure}

Four female undergraduate experimenters (average age: 21.2 years; standard deviation: 0.7 ) took part in the experiment, approaching individuals in each of the 3 definite conditions. The participants were randomly assigned. The first met participant was solicited in reference to the first condition script, the following one, in reference to the second condition script, and so on. In the "classic reciprocity" condition, the experimenter approached the passerby by saying, "Hello, I belong to a university association, and we are seeking to promote the University of South Brittany this Christmas season. We are giving away chocolate cake, would you like a piece?' After having accepted the piece of cake, the passerby was solicited for the target request: "Excuse me, would you watch my things ${ }^{2}$ while I go to the restroom? I won't be long." The experimenter then pointed to the public restroom that was located a couple of meters away. In the "generalized reciprocity" condition the participant was approached in the same manner as in the previous condition but was let go after taking a piece of cake. A few meters further, a second experimenter, supposedly not related to the operation but carrying several bags, approached the participant with the same target request. Finally in the control condition, this target request was presented to the participant the same way as in the previous conditions, except for the cake offer.

We hypothesized a linear increase in helping behavior across the conditions. More precisely, we envisioned "classic reciprocity" as statistically different from "generalized reciprocity," itself statistically different from the control condition.

\section{Results}

The data coming from the 4 experimenters not presenting a difference were aggregated in the analysis. A 2 (participant gender) X 3 (solicitation type) log-linear analysis was carried out on request acceptance. A main effect of solicitation type appears $\left(\chi^{2}(1,120)=34,761\right.$; $p=.0001 \varphi=.538)$. In accordance with our hypothesis, the 2 by 2 comparisons carried out on our data reveal that the "classic reciprocity" condition (37/40) produced a significantly higher acceptance rate than the "generalized reciprocity" condition $(28 / 40)\left(\chi^{2}(1,80)=6.65, \mathrm{p}<.01\right.$; $\varphi=.28)$; and a significantly higher rate than that of the

\footnotetext{
1 Participant age was not raised in order not the break the ecological context of the experiment.

2 Several bags containing the cakes to be distributed were laid out on the ground near the experimenter.
}

control condition $(13 / 40)\left(\chi^{2}(1,80)=30.72, \mathrm{p}<.0001\right.$, $\varphi=.53)$ (exact Fisher test $=p<.0001)$. In addition, the analyses reveal that "generalized reciprocity" produces significantly higher results than those in the control condition $\left(\chi^{2}(1,80)=11.26, \mathrm{p}<.001, \varphi=.35\right)$.

Table 1. Frequencies and percentages of acceptance of the request target in the different conditions

\begin{tabular}{lccc}
\hline Conditions & $\begin{array}{c}\text { Classic } \\
\text { Reciprocity }\end{array}$ & $\begin{array}{c}\text { Generalized } \\
\text { Recicprocity }\end{array}$ & Control \\
\hline Acceptance & $92.5 \% \mathrm{a}$ & $70 \% \mathrm{~b}$ & $32.5 \% \mathrm{c}$ \\
rates & $(37 / 40)$ & $(28 / 40)$ & $(13 / 40)$ \\
\hline
\end{tabular}

Distincts letters indicate a statistical difference at $\mathrm{p}<.001$ between conditions.

\section{Discussion}

Using the results of studies situated on the interface of the norm of reciprocity and the norm of social responsibility (Staub, 1972), the objective of our experiment was to investigate the existence of a broadened effect of the norm of reciprocity. In accordance with our hypothesis, the results reveal that an individual being offered a piece of cake is first of all, not only more likely to help the person who had previously given him the gift, but also more likely to help someone who had not been involved compared to a control situation where nothing was offered beforehand. These results replicate those of Regan (1971), Whatley and Al (1999), Boster and $A l$ (2001), Burger and $A l$ (2006) or Burger and $A l$ (2009) concerning the observed reciprocity effect between two individuals and support the proposal of Berkowitz and Daniels (1964) reiterated by Goranson and Berkowitz (1966), Greenglass (1969), Staub (1972) and Dieckman (2004) concerning the existence of a generalized reciprocity mechanism.

Compliance rates reported in our experimentation are particularly high, especially those observed in the classic and generalized reciprocity conditions (respectively $92.5 \%$ and $70 \%$ ). Christmas season, when the experimentation took place might explain these levels. Greenberg (2014), by analyzing amount of tips leaving in restaurants over two years, reveals that people tend to give more tips during the Christmas holydays than the rest of the year. According to the author, a social norm of generosity and altruism would be salient during this period.

The inconsistency in our data with those of Regan (1971) and Burger and $A l$ (2006, exp. 3) presenting a reciprocity effect at the exclusive benefit of the initial donor, could be explained by the specificity of the experimental situations put in place in these two studies. In Regan's (1971) experiment, the results are obtained on the basis of an initial gift given by the experimenter himself, which moreover justified this by the wish "to make the experience pleasant" (p. 631). Because of this donor's particular status and the context in which participating in the experiment was already the object of 
financial remuneration, this gift could have been seen by the participant as a complementary remuneration in kind, and thus inhibit a potential broadened effect toward the non-involved confederate. In Burger and Al's (2006, exp. 3) experiment, the experimental situation also moves away from a typical situation since the participant was solicited for the target request by a confederate other than the initial donor while he had just returned the favor to the latter. The absence of effect could then come from the previously returned favor.

In accordance with our hypothesis and with the results of Goranson and Daniels, (1964), Goranson and Berkowitz (1966) and Greenglass (1969), the generalized norm of reciprocity is shown to be statistically less influential than the classic norm of reciprocity. The visibility of the behavior, emphasizing it social evaluation could explain this result. Watley and $A l$ (1999), having demonstrated greater public rather than private reciprocity, consider the difference to be related to an individual's awareness of his own public image (Pendleton \& Batson, 1979; Baumeister, 1982), which has either been activated or not based on the condition. To the extent that the participant is truly the only one to know that he received a gift beforehand in a generalized reciprocity condition, such awareness would be less significant than in a classic reciprocity condition.

Another mechanism that our data do not make possible to dismiss remains in consideration. In parallel with investigations carried out on reciprocity and social responsibility, several studies have shown that receiving a gift (Levin \& Isen, 1975), finding coins (Levin \& Isen, 1975; Isen \& Simmonds, 1978), or being smiled at (Vrugt \& Vet, 2009) could put people in a good mood, capable of increasing the likelihood they would carry out acts of solicited or spontaneous helping behavior in turn (Guéguen $\&$ of Gail, 2003). It is not excluded that this mechanism also contributed to obtaining our results.

As this is an initial experimental attempt, there are certain limitations that future studies will need to consider. First, studies to come will need to take measures of affect in the different experimental conditions before making the target request in order to evaluate the relative weight of mood in the obtained effects. Also, in order to eliminate interpretation taken in the context of generalized reciprocity, the gift will need to be more disconnected from all considerations relative to assistance. As is, it cannot be excluded that accepting the cake was perceived by certain individuals as a way of helping to promote the university and despite our precautions, could have activated the norm of social responsibility. Finally, but in connection with the above, replication must be carried out in a period of the year less likely to promotes helping behaviors as this is the case during the Christmas period.

In addition to these limitations, the present experiment contributes elements of knowledge related to the implementation. While Cialdini (2004) cites many examples of reciprocity in real life, the majority of research has been carried out in laboratories and with students. This experiment carried out in the street and with passersby demonstrates that the effect is sufficiently robust to be observed in a context however favorable to the occurrence of parasitic variables. Moreover, it suggests that whatever the experimental condition considered and whatever the theoretical interpretation selected, the preliminary distribution of a low value gift is an effective tool at increasing subsequent submission of individuals to a pro-social request.

\section{References}

Batson, C.D., Coke, J.S., Chard, F., Smith, D., \& Taliaferro, A. (1979) Generality of the "glow of goodwill": The effect of mood on helping and information acquisition. Social Psychology Quarterly, 42, 176-179.

Baumeister, R.F. (1982). A self-presentational view of social phenomena. Psychological Bulletin, 91, 3-26.

Berkowitz, L., \& Daniels, L.R. (1963). Responsibility and dependency. Journal of Abnormal and Social Psychology, 66, 429-436.

Berkowitz, L., \& Daniels, L.R. (1964). Affecting the salience of the social responsibility norm: Effects of past help on the response to dependency relationships. Journal of Abnormal and Social Psychology, 68, 275-281.

Boster, F.J., Fediuk, T.A., \& Kotowski, M.J. (2001). The effectiveness of an altruistic appeal in the presence and absence of favors. Communication Monographs, 68, 340-346.

Burger, J.M., Ehrlichman, A.M., Raymond, N.C., Ishikawa, J.M., \& Sandoval, J. (2006) Reciprocal favor exchangeand compliance. Social Influence, 1, 169-184.

Burger, J.M., Horita, M., Kinoshita, L., Roberts, K., \& Vera, C. (1997) Effects of time on the norm of reciprocity. Basic and Applied Social Psychology, 19, 91-100.

Burger, J.M., Sanchez, J., Imberi, J.E., \& Grande, L.R. (2009). The norm of reciprocity as an internalized social norm: Returning favors even when no one finds out. Social Influence, 4, 11-17.

Cialdini, R B., (2004). Influence et Manipulation. Paris, First Clark \& Waddell, 1986

Cialdini, R.B., Vincent, J.E., Lewis, S.K., Catalan, J., Wheeler, D., \& Darby, B.L. (1975). Reciprocal Concessions Procedure for Inducing Compliance: The door-in the face Technique. Journal of Personnality and Social Psychology, 31, 206-215.

Diekmann, A. (2004). The power of reciprocity: Fairness, reciprocity, and stakes in variants of the dictator game. Journal of conflict resolution, 48, 487-505.

Dillard, J.P., Hunter, J.E., \& Burgoon, M. (1984). Sequential-request persuasive strategies. Human Communication Research, 10, 461-488.

Dovidio, J.F., Piliavin, J.A., Schroeder, D.A., \& Penner, L.A. (2006). The Social Psychology of Prosocial Behavior. Mahwah, N.J.: Lawrence Erlbaum Associates.

Goranson, R.E., \& Berkowitz, L. (1966). Reciprocity and responsibility reactions to prior help. Journal of Personality and Social Psychology, 3, 227-232.

Gouldner, A.W. (1960). The norm of reciprocity: A preliminary statement American Sociological Review, 25, 161-178.

Greenberg, A.E. (2014). On the complementarity of prosocial norms: The case of restaurant tipping during the holidays. Journal of Economic Behavior \& Organization, 97, 103-112.

Greenglass, E.R. (1969). Effect of prior help and hindrance on willingness to help another: reciprocity or social responsibility. Journal of Personality and Social Psychology. 11, 224-231.

Guéguen, N., \& de Gail, M.A. (2003). The effect of smiling on helping behavior: Smiling and Good Samaritan behavior. Communication Reports, 16, 133-140.

Harris, M.B., \& Smith, R.J. (1975). Mood and Helping. The Journal of Psychology 91, 215-221.

Horowitz, I.A. (1968). Effect of choice and locus of dependence on helping behavior. Journal of Personality and Social Psychology, 8, 373-376.

Isen, A.M., \& Levin, P. (1972). Effect of feeling good on helping: cookies and kindness. Journal of Personality and Social Psychology, 21, 384-388. 
Isen, A.M., Shalker, T.E., Clark, M., \& Karp, L. (1978). Affect, accessibility of material in memory, and behavior: A cognitive loop? Journal of Personality and Social Psychology, 36, 1-12.

Levin, P.R., \& Isen, A.M. (1975). Further studies on the effect of feeling good on helping. Sociometry, 38, 141-147.

Pendleton, M.G., \& Batson, C.D., 1979. Self-presentation and the door-inthe-face technique for inducing compliance. Personality and Social Psychology Bulletin 5, 77-81.

Regan, D.T. (1971). Effects of a favor and liking on compliance. Journal of Experimental Social Psychology, 7, 627-639.

Solomon, H., Zener Solomon, L., Arnone, M.M., Maur, B.J., Reda, R.M., \& Roth, E.O. (1981). Anonymity and helping. Journal of Social Psychology, 113, 37-43.

Schwartz, S.H., \& Howard, J.A. (1981). A normative decision-making model of altruism. In: J.P. Rushton \& R.M. Sorrentino (Eds.),
Altruism and helping behavior (pp. 89-211). Hillsdale, NJ: Erlbaum.

Staub, E. (1972). Instigation to goodness: The role of social norms and interpersonal influence, Journal of Social Issues, 28, 131-150.

Terrier, L., Marfaing, B., \& Boldi, M-O. (2013). Door-in-the-face: Is it really necessary that both requests be made by the same requester? Psychological Reports, 113, 1-8.

Vrugt, A., \& Vet., C. (2009). Effects of a smile on mood and helping behavior. Social Behavior and Personality, 37, 1251-1258.

Warneken, F. (2013c). The development of altruistic behavior: Helping in children and chimpanzees. Social Research, 80, 431-442.

Whatley, M.A., Webster, J.M., Smith, R.H., \& Rhodes, A. (1999). The effect of a favor on public and private compliance: How internalized is the norm of reciprocity? Basic and Applied Social Psychology, $21,251-259$. 